SUPPORTING INFORMATION

The susceptibility of an airborne common cold virus to relative humidity

Sadegh Niazi ${ }^{\dagger}$, Robert Groth ${ }^{\dagger}$, Luke Cravigan ${ }^{\dagger}$, Congrong He ${ }^{\dagger}$, Julian W Tang ${ }^{\ddagger}$, Kirsten Spann ${ }^{\S}$, Graham R. Johnson ${ }^{\dagger *}$

${ }^{\dagger}$ Queensland University of Technology (QUT), Science and Engineering Faculty, School of Earth and Atmospheric Sciences, Brisbane, Australia.

${ }^{\ddagger}$ Clinical Microbiology, University Hospitals Leicester, Level 5 Sandringham Building, Leicester Royal Infirmary, Infirmary Square, Leicester LE1 5WW, UK.

${ }^{\dagger}$ Queensland University of Technology, Faculty of Health, School of Biomedical Sciences, Brisbane, Australia.

*Corresponding author: Graham R. Johnson; E-mail: g.johnson@qut.edu.au 
Figure S1: Experimental process.

Figure S2: Sample timing.

Figure S3: Schematic diagram of the applied H-TDMA instruments.

Figure S4: Schematic and photograph of the rotating chamber and instrument interfaces.

Text I: Temperature and RH maintenance and measurement.

Text II: Aerosol Production.

Text III: Aerosol Sampling.

Text IV: HRV-16 Propagation.

Table S1: The amount of extracted RNA (ng/ml) and TCID $50 / \mathrm{mL}$ for each experiment at $\mathrm{RH}_{<\mathrm{E}}, \mathrm{RH}_{\mathrm{E}-\mathrm{D}}$ and $\mathrm{RH}>\mathrm{D}$.

Table S2: The amount of extracted RNA ( $\mathrm{ng} / \mathrm{ml})$ and $\mathrm{TCID}_{50} / \mathrm{mL}$ for each experiment of effloresced (treatment) and deliquesced (control-treatment) states.

Table S3: One-way ANOVA results of surviving fraction at different aging times for $\mathrm{RH}_{<\mathrm{E}}$, $\mathrm{RH}_{\mathrm{E}-\mathrm{D}}$ and $\mathrm{RH}>_{\mathrm{D}}$.

Table S4: One-way ANOVA results of surviving fraction at different aging times for effloresced (treatment) and deliquesced (control-treatment) states.

Figure S5: HRV-16 survival from vibrating mesh nebulizer testing before and after aerosolization. 
Phase 1- Hygroscopic characterization (locating the hysteresis zone)

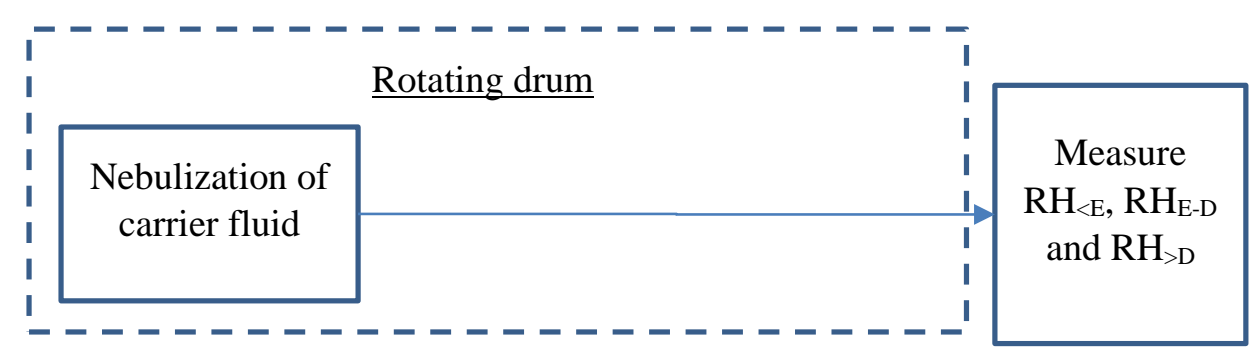

Phase 2 - Virus decay in the $\mathrm{RH}_{<\mathrm{E}}, \mathrm{RH}_{\mathrm{E}-\mathrm{D}}$ and $\mathrm{RH}_{>_{\mathrm{D}}}$ zones without Pre-treatment

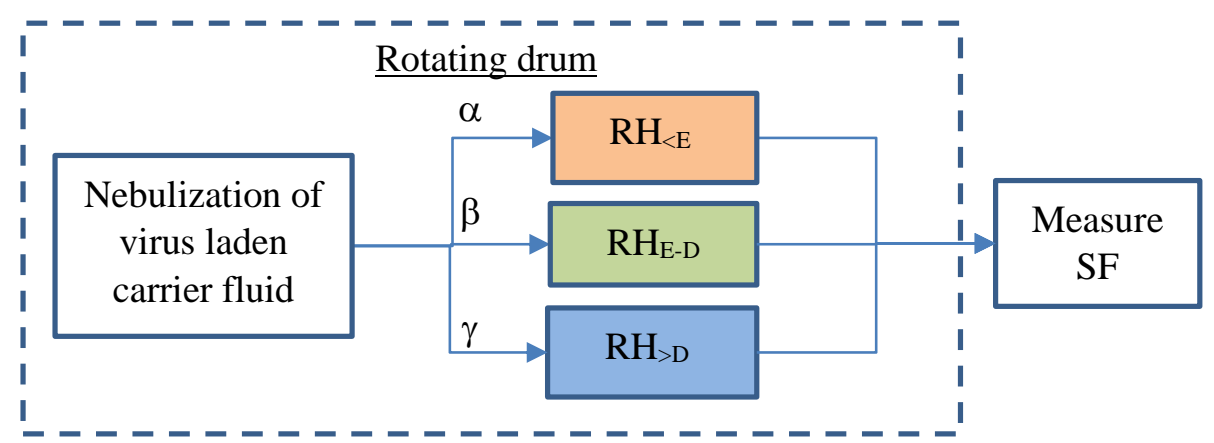

Phase 3 - Virus decay in $\mathrm{RH}_{\mathrm{E}-\mathrm{D}}$ zone in the Effloresced and Deliquesced (Control) States.

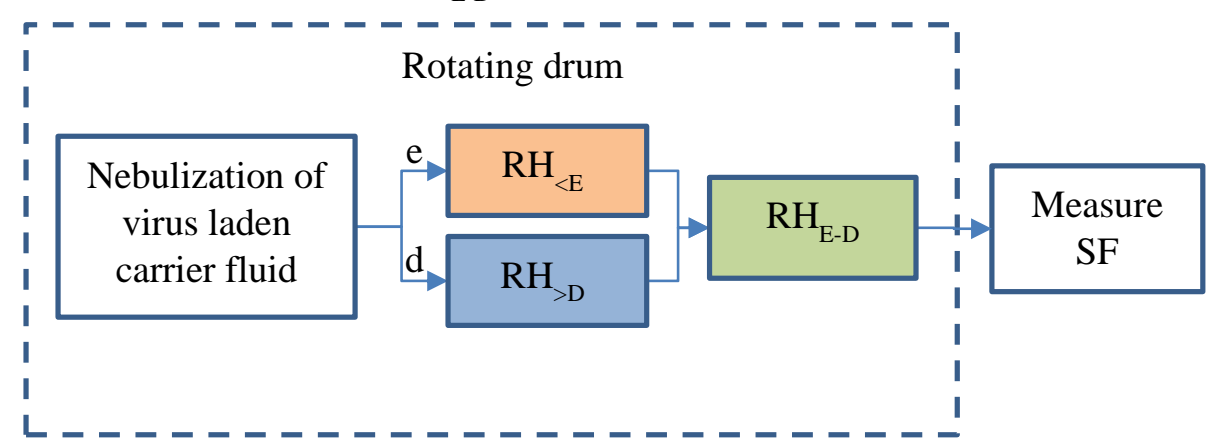

Figure S1: Experimental process. Labels " $\alpha, \beta$ and $\gamma$ " denote phase 2 experiments. Labels "e" and "d" denote phase 3 experiments. 


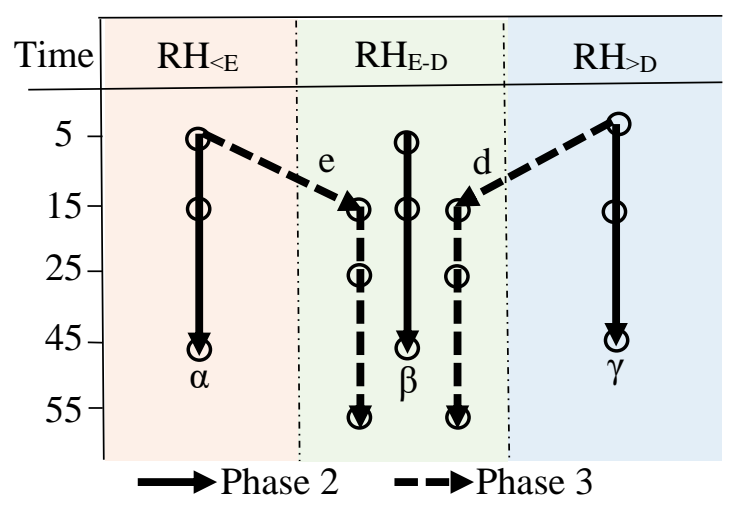

Figure S2: Sample timing. Sample ages at the times of extraction are indicated by the symbol "O". Phase 2 samples are linked by continuous lines labelled " $\alpha, \beta$ and $\gamma$ ". Phase 3 by dashed lines labelled "e" and "d". Samples were collected and SF determined at aerosol age timepoints of 5, 15 and 45 minutes for phase 2 and 5, 15, 25 and 55 minutes for phase 3. 


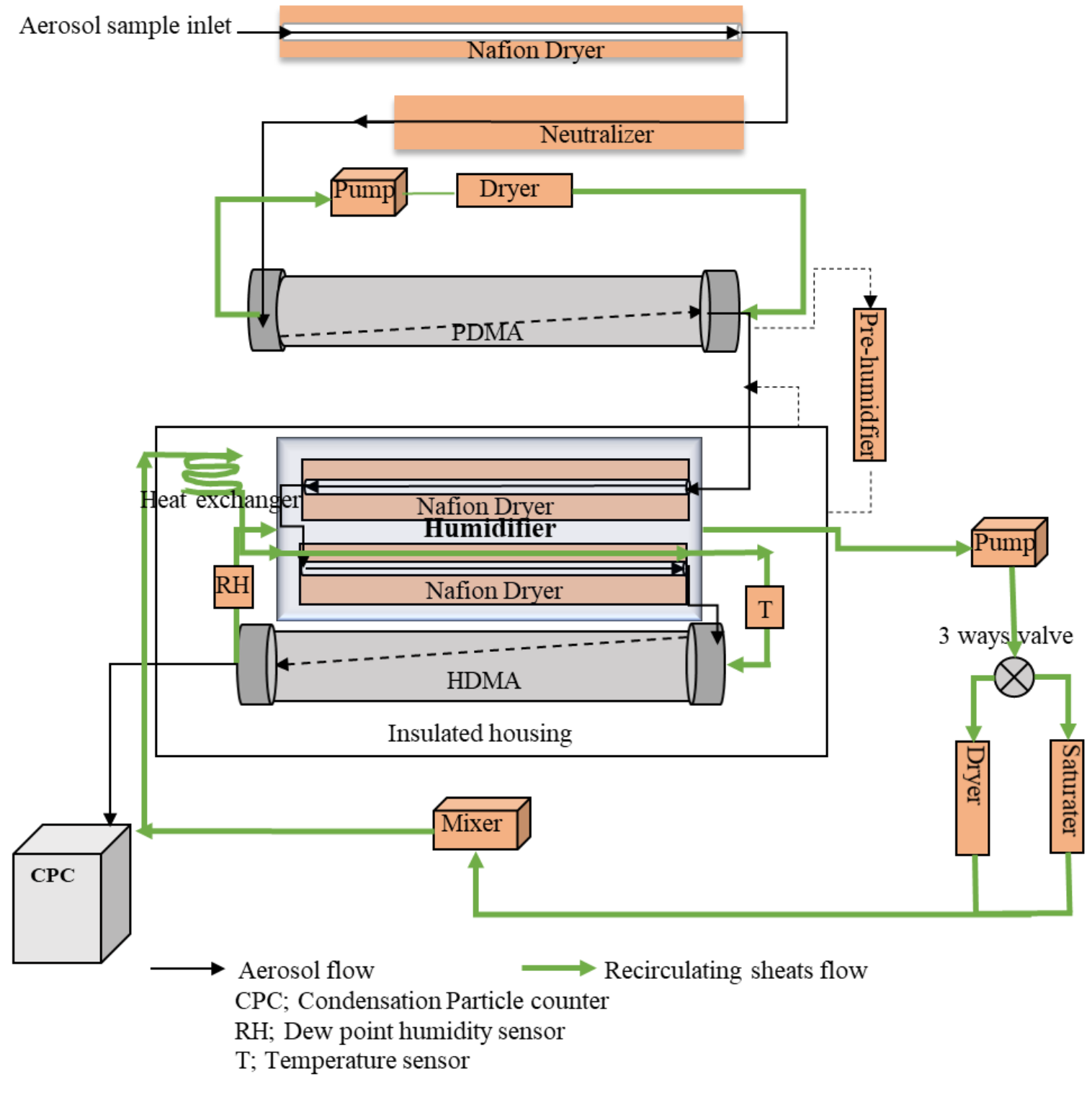

Figure S3: Schematic diagram of the applied H-TDMA instruments. 


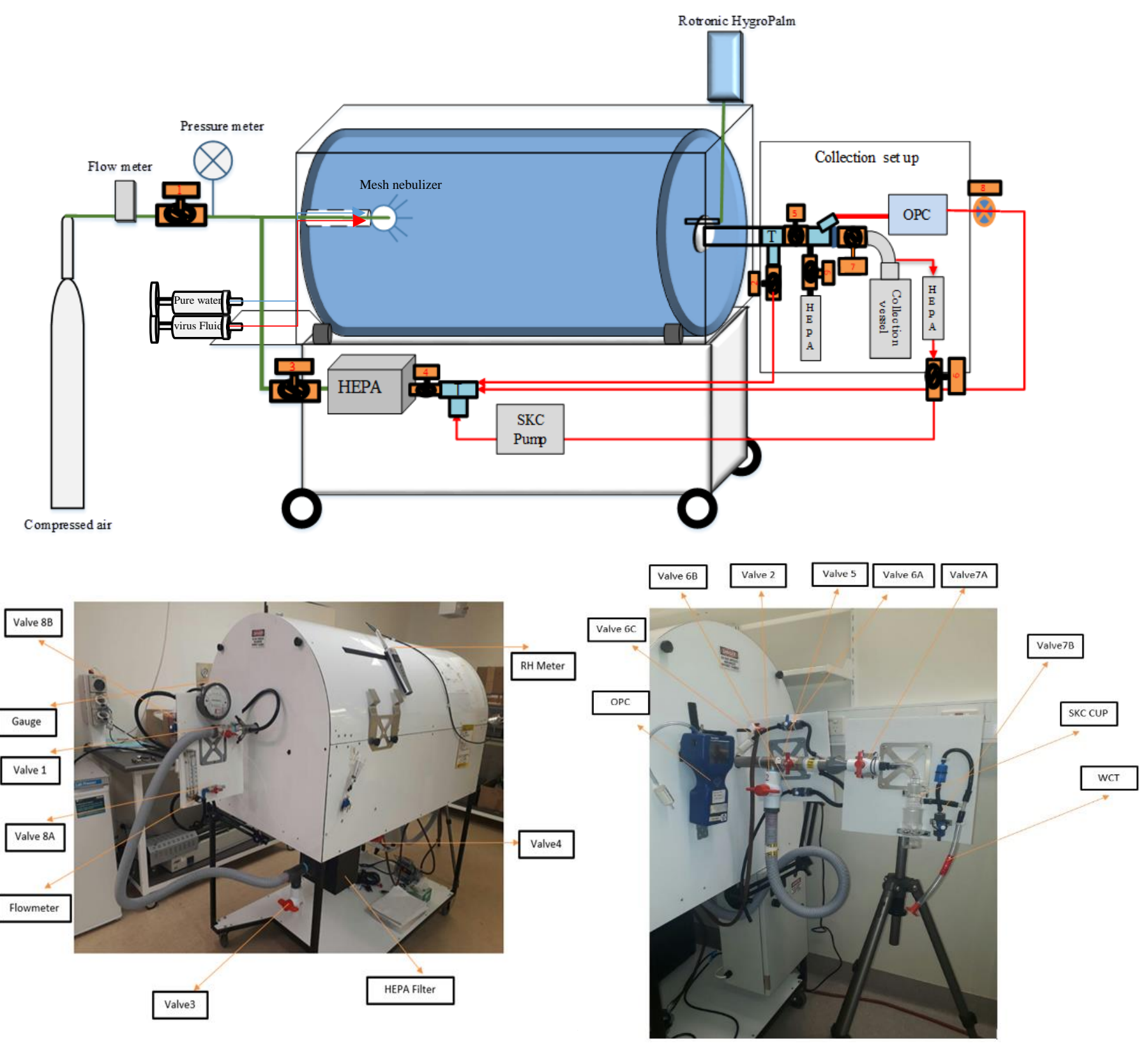

Figure S4: Schematic and photograph of the rotating chamber and instrument interfaces (rotator, valves and connectors). WCT; water collection tube, OPC; Optical Particle Counters. 


\section{Text I}

Temperature control was achieved using a thermoelectric heating and cooling system embedded in the wall of the outer enclosure and controlled by a proportional integral derivative (PID) feedback system connected to a temperature sensor inside the outer enclosure. This allowed for internal temperature adjustment within $\pm 0.5^{\circ} \mathrm{C}$, for the range $13-30{ }^{\circ} \mathrm{C}$. A Rotronic HygroPalm - HP23-A with 1 minute time interval was used to measure RH and temperature inside the drum. For measuring the virus survival in an effloresced state, virus suspension was first nebulized inside the system over a period of 5 minutes, starting from a very low RH (6\%). The water component of the nebulized aerosol increased the $\mathrm{RH}$ to $33 \%$ (still less than the efflorescence RH measured for the aerosol). A small amount of ultrapure nuclease free water purified by membrane filtration was then nebulized over a period of 5 minutes to bring the system into the hysteresis $\mathrm{RH}$ range at approximately $57 \%$ of $\mathrm{RH}$. To test virus survival in a deliquesced (droplet) state, water was first nebulized to bring the system to $80 \%$ and the virus suspension was then nebulized over a period of 5 minutes, bringing the system up to $90.4 \%$ $\mathrm{RH}$, which is well above the deliquescence RH measured for the aerosol. Dry air was then injected over a period of 5 minutes after nebulization to reduce the $\mathrm{RH}$ back to a hysteresis range value of $57 \%$. 


\section{Text II}

The novel use of vibrating mesh nebulizer was chosen over the more traditional Collison nebulizer in the current application because it offered a very high fluid nebulization rate (1.5 $\mathrm{mL} / \mathrm{min}$ ) without the need for a carrier air flow, allowing better temporal resolution in time sensitive measurements. The nebulizer employs an electroformed plate perforated with conical holes and mounted on a piezoelectric ceramic ring that causes the plate to vibrate in response to an applied alternating potential difference. The vibrating mesh draws fluid through the holes to generate aerosols without the need for a carrier flow ${ }^{1}$. The plate used in the nebulizer was 5 $\mathrm{mm}$ in diameter, pierced with 1000 tapered holes and vibrated at a frequency of $128 \mathrm{kHz}$ to produce a unimodal droplet size distribution with diameters between 1 and $5 \mu \mathrm{m}$ in diameter ${ }^{2}$. 


\section{Text III}

The aerosols were extracted from the drum using an SKC Biosampler with $5 \mathrm{ml}$ collection vessels (\#225-9593, SKC). The BioSampler is an improved impinger-type air sampler consisted of a $5 \mathrm{ml}$ collection vessel, connecting tube and a pump with the flow rate of $12.5 \mathrm{~L}$ $\min ^{-1}$ and collects aerosols using a swirling flow of liquid media (PBS by drawing air through three $0.630 \mathrm{~mm}$ tangential sonic nozzles ${ }^{3}$. 


\section{Text IV}

HRV-16 was grown in Ohio HeLa cells in VP-SFM (Life Technologies, USA). Once 80\% cell death was achieved, cell culture supernatant and remaining cells were collected and centrifuged at $2000 \times g$ to form cell pellets. Supernatant was removed and the pellets were freeze-thawed twice to disrupt cell membranes and release bound viral particles, then clarified by centrifugation at $2000 \times \mathrm{g}$. This clarified preparation and the original cell supernatant were combined, then the virus was concentrated by centrifugation through $15 \mathrm{~mL}$ spin columns (size cut-off $0.2 \mu \mathrm{m})\left(\right.$ Merc Millipore Amicon Ultra) at $2500 \times \mathrm{g}$ for $25 \mathrm{~min}$ at $4{ }^{\circ} \mathrm{C}$. The concentrated virus was quantified using a tissue culture infectious dose $\left(\mathrm{TCID}_{50}\right)$ assay, and stored at $-80{ }^{\circ} \mathrm{C}$. 
Table S1: The amount of extracted RNA (ng/ml) and TCID $50 / \mathrm{mL}$ for each experiment at $\mathrm{RH}_{<\mathrm{E}}, \mathrm{RH}_{\mathrm{E}-\mathrm{D}}$ and $\mathrm{RH}>\mathrm{D}$.

\begin{tabular}{|l|l|l|l|l|l|l|l|}
\hline \multirow{2}{*}{ experiment } & $\begin{array}{l}\text { Aging } \\
\text { time }\end{array}$ & \multicolumn{2}{|c|}{$\mathrm{RH}_{<\mathrm{E}}$} & \multicolumn{2}{c|}{$\mathrm{RH}_{\mathrm{E}-\mathrm{D}}$} & \multicolumn{2}{c|}{$\mathrm{RH}_{>\mathrm{D}}$} \\
\hline \multirow{5}{*}{1} & $\begin{array}{l}\text { RNA } \\
(\mathrm{ng} / \mathrm{ml})\end{array}$ & $\begin{array}{l}\text { Measured } \\
\text { TCID } 50\end{array}$ & $\begin{array}{l}\text { RNA } \\
(\mathrm{ng} / \mathrm{ml})\end{array}$ & $\begin{array}{l}\text { TCID } \\
\text { /ml }\end{array}$ & $\begin{array}{l}\text { RNA } \\
(\mathrm{ng} / \mathrm{ml})\end{array}$ & $\begin{array}{l}\text { TCID }_{50} \\
\text { /ml }\end{array}$ \\
\cline { 2 - 8 } & Before & 37657.14 & 12500000 & 42000 & 93700000 & 38685.82 & 52500000 \\
\cline { 2 - 8 } & 5 minutes & 3085.71 & 167000 & 3045.72 & 395000 & 2754.28 & 1250000 \\
\cline { 2 - 8 } & $\begin{array}{c}15 \\
\text { minutes }\end{array}$ & 2685.73 & 125000 & 3005.71 & 395000 & 2634.34 & 527000 \\
\cline { 2 - 8 } & $\begin{array}{c}45 \\
\text { minutes }\end{array}$ & 2257.14 & 52700 & 2257.16 & 225000 & 2474.29 & 39500 \\
\hline \multirow{5}{*}{2} & Before & 39371.42 & 22200000 & 42457.14 & 93700000 & 38857.15 & 52500000 \\
\cline { 2 - 8 } & 5 minutes & 2828.57 & 525000 & 2811.42 & 703000 & 2800 & 1250000 \\
\cline { 2 - 8 } & $\begin{array}{c}15 \\
\text { minutes }\end{array}$ & 2822.85 & 395000 & 2800.16 & 395000 & 2817.14 & 937000 \\
\cline { 2 - 8 } & $\begin{array}{c}45 \\
\text { minutes }\end{array}$ & 2514.28 & 296000 & 2725.71 & 12500 & 2702.86 & 125000 \\
\hline \multirow{5}{*}{3} & Before & 28514.28 & 2960000 & 42405.73 & 93700000 & 39028.57 & 52500000 \\
\cline { 2 - 8 } & 5 minutes & 2514.28 & 52500 & 2788.58 & 395000 & 3668.57 & 1250000 \\
\cline { 2 - 8 } & $\begin{array}{c}15 \\
\text { minutes }\end{array}$ & 2628.57 & 39500 & 2668.57 & 125000 & 3280 & 937000 \\
\cline { 2 - 8 } & $\begin{array}{c}45 \\
\text { minutes }\end{array}$ & 2457.14 & 29600 & 2657.14 & 62700 & 2702.85 & 39500 \\
\hline
\end{tabular}


Table S2: The amount of extracted RNA ( $\mathrm{ng} / \mathrm{ml})$ and TCID $_{50} / \mathrm{mL}$ for each experiment of effloresced (treatment case) and deliquesced (control-treatment case) states.

\begin{tabular}{|c|c|c|c|c|c|}
\hline experiment & $\begin{array}{l}\text { Aging } \\
\text { time }\end{array}$ & $\begin{array}{l}\text { Efflorescec } \\
\text { case }\end{array}$ & treatment & $\begin{array}{l}\text { Deliquesc } \\
\text { treatment) }\end{array}$ & $\begin{array}{l}\text { (control- } \\
\text { ase }\end{array}$ \\
\hline \multirow{5}{*}{1} & & $\begin{array}{l}\text { RNA } \\
(\mathrm{ng} / \mathrm{ml})\end{array}$ & $\begin{array}{l}\text { Measured } \\
\text { TCID }_{50}\end{array}$ & $\begin{array}{l}\text { RNA } \\
(\mathrm{ng} / \mathrm{ml})\end{array}$ & $\begin{array}{l}\text { Measured } \\
\text { TCID }_{50}\end{array}$ \\
\hline & Before & 26651.43 & 703000 & 36742.81 & 22200000 \\
\hline & $\begin{array}{l}15 \\
\text { minutes }\end{array}$ & 3040 & 12500 & 1942.85 & 395 \\
\hline & $\begin{array}{l}25 \\
\text { minutes }\end{array}$ & 2994.26 & 9370 & 2114.28 & 222 \\
\hline & $\begin{array}{l}55 \\
\text { minutes }\end{array}$ & 2990 & 5270 & 1428.57 & 16 \\
\hline \multirow{4}{*}{2} & Before & 44400 & 29600000 & 36742.8 & 22200000 \\
\hline & $\begin{array}{l}15 \\
\text { minutes }\end{array}$ & 3617.14 & 325000 & 1650 & 10395 \\
\hline & $\begin{array}{l}25 \\
\text { minutes }\end{array}$ & 2914.28 & 125000 & 1500 & 3937 \\
\hline & $\begin{array}{l}55 \\
\text { minutes }\end{array}$ & 2691.42 & 7030 & 1465 & 3076 \\
\hline \multirow{4}{*}{3} & Before & 44400 & 52500000 & 36742.81 & 22200000 \\
\hline & $\begin{array}{l}15 \\
\text { minutes }\end{array}$ & 3251.43 & 837000 & 1885.71 & 3600 \\
\hline & $\begin{array}{l}25 \\
\text { minutes }\end{array}$ & 2622.86 & 527000 & 834.22 & 1500 \\
\hline & $\begin{array}{l}55 \\
\text { minutes }\end{array}$ & 2680 & 125000 & 1428.51 & 800 \\
\hline
\end{tabular}


Table S3: One-way ANOVA results of surviving fraction at different aging times for $\mathrm{RH}_{<\mathrm{E}}$, $\mathrm{RH}_{\mathrm{E}-\mathrm{D}}$ and $\mathrm{RH}>\mathrm{D}(* 0.033, * * 0.002$ and $* * * 0.001)$.

\begin{tabular}{|c|c|c|c|c|}
\hline $\begin{array}{l}\text { Tukey's multiple comparisons test of SFs } \\
\text { between sampling times. }\end{array}$ & Mean Diff. & Significant? & Summary & $\begin{array}{l}\text { Adjusted P } \\
\text { Value }\end{array}$ \\
\hline $5 \min \mathrm{RH}_{<\mathrm{E}}$ versus $5 \min \mathrm{RH}_{\mathrm{E}-\mathrm{D}}$ & 0.15 & Yes & $*$ & 0.03 \\
\hline $5 \min \mathrm{RH}_{<\mathrm{E}}$ versus $5 \min \mathrm{RH}_{>\mathrm{D}}$ & -0.07 & No & $\mathrm{ns}$ & 0.7 \\
\hline $5 \min \mathrm{RH}_{\mathrm{E}-\mathrm{D}}$ versus $5 \min \mathrm{RH}_{>_{\mathrm{D}}}$ & -0.22 & Yes & $* * *$ & $<0.001$ \\
\hline $15 \min \mathrm{RH}_{<\mathrm{E}}$ versus $15 \mathrm{~min} \mathrm{RH}_{\mathrm{E}-\mathrm{D}}$ & 0.12 & No & $\mathrm{ns}$ & 0.1 \\
\hline $15 \mathrm{~min} \mathrm{RH}_{<\mathrm{E}}$ versus $15 \mathrm{~min} \mathrm{RH}_{>\mathrm{D}}$ & -0.02 & No & $\mathrm{ns}$ & $>0.999$ \\
\hline $15 \min \mathrm{RH}_{\mathrm{E}-\mathrm{D}}$ versus $15 \mathrm{~min} \mathrm{RH}_{>\mathrm{D}}$ & -0.15 & Yes & $*$ & 0.03 \\
\hline $45 \mathrm{~min} \mathrm{RH}_{<\mathrm{E}}$ versus $45 \mathrm{~min} \mathrm{RH}_{\mathrm{E}-\mathrm{D}}$ & 0.11 & No & ns & 0.2 \\
\hline $45 \mathrm{~min} \mathrm{RH}_{<\mathrm{E}}$ versus $45 \mathrm{~min} \mathrm{RH}_{>\mathrm{D}}$ & 0.11 & No & ns & 0.2 \\
\hline $45 \min \mathrm{RH}_{\mathrm{E}-\mathrm{D}}$ versus $45 \min \mathrm{RH}_{>\mathrm{D}}$ & 0.0001 & No & $\mathrm{ns}$ & $>0.999$ \\
\hline $5 \min \mathrm{RH}_{<\mathrm{E}}$ versus $15 \min \mathrm{RH}_{<\mathrm{E}}$ & 0.053 & No & $\mathrm{ns}$ & 0.9 \\
\hline $5 \min \mathrm{RH}_{<\mathrm{E}}$ versus $45 \mathrm{~min} \mathrm{RH}_{<\mathrm{E}}$ & 0.099 & No & $\mathrm{ns}$ & 0.3 \\
\hline $15 \mathrm{~min} \mathrm{RH}_{<\mathrm{E}}$ versus $45 \mathrm{~min} \mathrm{RH}_{<\mathrm{E}}$ & 0.045 & No & $\mathrm{ns}$ & 0.9 \\
\hline $5 \min \mathrm{RH}_{\mathrm{E}-\mathrm{D}}$ versus $15 \min \mathrm{RH}_{\mathrm{E}-\mathrm{D}}$ & 0.026 & No & $\mathrm{ns}$ & 0.9 \\
\hline $5 \min \mathrm{RH}_{\mathrm{E}-\mathrm{D}}$ versus $45 \min \mathrm{RH}_{\mathrm{E}-\mathrm{D}}$ & 0.051 & No & $\mathrm{ns}$ & 0.9 \\
\hline $15 \min \mathrm{RH}_{\mathrm{E}-\mathrm{D}}$ versus $45 \min \mathrm{RH}_{\mathrm{E}-\mathrm{D}}$ & 0.03 & No & $\mathrm{ns}$ & 0.9 \\
\hline $5 \min \mathrm{RH}_{>\mathrm{D}}$ versus $15 \min \mathrm{RH}_{>\mathrm{D}}$ & 0.1 & No & $\mathrm{ns}$ & 0.3 \\
\hline $5 \min \mathrm{RH}_{>\mathrm{D}}$ versus $45 \min \mathrm{RH}_{>\mathrm{D}}$ & 0.3 & Yes & $* * *$ & $<0.001$ \\
\hline $15 \min R_{D}$ versus $45 \min R H_{>D}$ & 0.2 & Yes & $* *$ & 0.008 \\
\hline
\end{tabular}


Table S4: One-way ANOVA results of surviving fraction at different aging times for Effloresced (treatment case) and deliquesced (control-treatment case) states $(* 0.033, * *$ 0.002 and $* * * 0.001)$.

\begin{tabular}{|l|r|l|l|c|}
\hline Tukey's multiple comparisons test & Mean Diff. & Significant? & Summary & Adjusted P Value \\
\hline $\begin{array}{l}\text { Surviving fraction of 15 minutes effloresced } \\
\text { state versus 15 minutes deliquesced state }\end{array}$ & 0.16 & Yes & $* * *$ & $<0.001$ \\
\hline $\begin{array}{l}\text { Surviving fraction of 25 minutes effloresced } \\
\text { state versus 25minutes deliquesced state }\end{array}$ & 0.11 & Yes & $* * *$ & $<0.001$ \\
\hline $\begin{array}{l}\text { Surviving fraction of 55 minutes effloresced } \\
\text { case versus 55 minutes deliquesced state }\end{array}$ & 0.03 & No & ns & 0.72 \\
\hline $\begin{array}{l}\text { Surviving fraction of 15 minutes versus 25 } \\
\text { minutes effloresced state }\end{array}$ & 0.05 & No & ns & 0.36 \\
\hline $\begin{array}{l}\text { Surviving fraction of 15 minutes versus 55 } \\
\text { minutes effloresced state }\end{array}$ & 0.13 & Yes & $* *$ & 0.002 \\
\hline $\begin{array}{l}\text { Surviving fraction of 15 minutes versus 25 } \\
\text { minutes deliquesced state }\end{array}$ & 0.002 & No & ns & 0.999 \\
\hline $\begin{array}{l}\text { Surviving fraction of 15 minutes versus 55 } \\
\text { minutes deliquesced state }\end{array}$ & 0.003 & No & ns & $>0.999$ \\
\hline
\end{tabular}




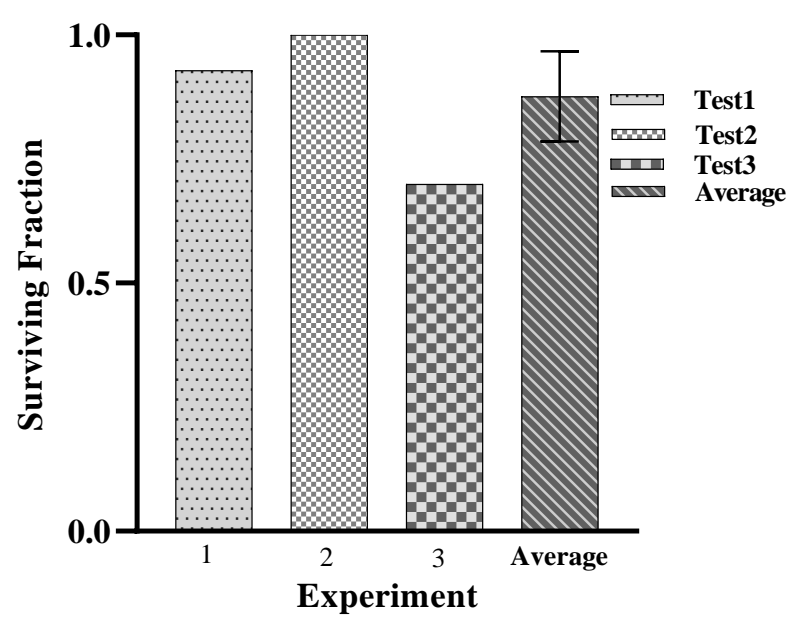

Figure S5: HRV-16 survival from vibrating mesh nebulizer testing before and after aerosolization (Results represent three experimental replicates and their average). Mean Surviving Fraction $(\mathrm{SF})=0.87 \pm 0.15$ 


\section{Q REFERENCES}

(1) Ghazanfari, T.; Elhissi, A. M.; Ding, Z.; Taylor, K. M., The influence of fluid physicochemical properties on vibrating-mesh nebulization. Int J Pharm 2007, 339, 103-11.

(2) Astudillo, A.; Leung, S. S. Y.; Kutter, E.; Morales, S.; Chan, H. K., Nebulization effects on structural stability of bacteriophage PEV 44. European journal of pharmaceutics and biopharmaceutics : official journal of Arbeitsgemeinschaft fur Pharmazeutische Verfahrenstechnik e.V 2018, 125, 124130.

(3) Willeke, K.; Lin, X.; Grinshpun, S. A., Improved aerosol collection by combined impaction and centrifugal motion. Aerosol Science and Technology 1998, 28, 439-456. 